mind. A few days later I observed a swelling on the right side of the neck about in the middle of the sterno-mastoid muscle. On palpation there was a good-sized packet of soft lymph-nodes on both sides of the sterno-mastoid muscle. In addition there were numerous discrete lymph-nodes in the posterior and lower portion of the same side of the neck. The skin was normal and not adherent over the swelling and the lymph-nodes were movable. He complained of some pain when I palpated the neck or moved his head. The temperature was not elevated at this time; the tongue was somewhat coated. The tonsils were slightly enlarged and clean and the mucous membrane of the soft palate and pharynx was reddened. A culture from the throat showed a growth on blood serum of streptococcus, but I could not reconcile the absence of symptoms with a streptococcic infection capable of producing enlarged lymph-nodes. In addition, the enlargement was not at the angle of the jaw, but there was a distinct space between it and the enlarged packet of glands. Two or three days later the lymph-nodes enlarged on the left side of the neck, but not to the same extent. The character and location of the swelling were similar on both sides. After about a week the swelling subsided rapidly to a certain point and then diminished slowly, so that some enlargement could be detected at the end of four weeks. During this time the child was quite pale and became fatigued rather easily, otherwise he seemed to feel as usual.

About one week later I was called to one of my patients, a boy seven years old, with a similar swelling on one side of his neck. His temperature was $101^{\circ} \mathrm{F}$. and he complained of tenderness in the neck when handled and of pain when he moved his head. Swallowing also caused some pain. These symptoms together with some maaise began on the day previous. The throat was slightly reddened. A culture on serum showed no pathogenic organisms. On the following day his fever had subsided and the tenderness was less marked. On the fourth day he was allowed to go out because he felt so much better. The swelling persisted for several days, but affected only one side.

The mothers of two other patients of mine reported similar swellings in the neck, but the subjective symptoms were so slight that I was not asked to see them. Since then I have heard of several other cases in Boston and its vicinity. In two of these cases the disease was not of so mild a type as those which I have described. One of these children was confined to the house for two weeks and the fever persisted for several days.

This brief description may be of assistance to those who are not familiar with the disease.

REFERENCES.

Pfeiffer, E.: Drusenfieber, Jahrbuch f. Kinderheilk, Bd. xxix, s. 257,1889 .

(An appendix to Pfeiffer's article.

Starck: Über das Drüsenfieber, Jahrbuch f. Kinderheilk, Bd. xxxi, s. 454, 1890

Rauchfuss, C.: Zur Casuistik des Drüsenfiebers, Jahrbuch f inderheilk, Bd. xxxi, s. 461, 1890.

Filatow Nil. Vorlesungen iu. Acute Infections-Krankheiten im Kindersalter, s. 572. Wien, 1897.

\section{REPORT ON EARLY DIAGNOSIS OF TUBERCULOSIS.*}

BY DR8, A. C. KLEB8, CHICAGO; J. H. MUBSER, PHILADELPHIA; F. BILlings, Chicago; J. C. Wilson, philadelphia; H. R. M. LANDI8, PHILADELPHIA.

THE diagnosis of phthisis, of that well-developed stage of pulmonary tuberculosis, offers no difficulty whatever, the symptoms are well marked and typical, the pulmonary signs easily discoverable, the expectoration contains bacilli; the clinical picture is so typical that mistakes in diagnosis cannot well be made, even by the superficially trained observer. But this stage of pathological development in the great majority of cases is reached only after very long periods, during which all signs and symptoms are less typical, less marked from that time on when infection took place. During this time, which marks the true incipiency of the malady and which anatomically is characterized by the formation of few isolated tubercles in lymph glands or lung tissue, no or only vague general symptoms exist, none on which to base a positive diagnosis of the disease. Investigation has shown that such infections are of very frequent occurrence, and that only in a comparatively small percentage of these cases does the disease develop further. However, this percentage is sufficiently large to make tuberculosis the most destructive of all diseases, and the demonstration of its frequent and spontaneous arrest and of its curability by certain therapeutic measures at an early period of its development, must induce efforts of recognition long before the stage of phthisis is reached.

The discovery of the tubercle bacillus in the sputum of patients suffering from the disease constitutes a diagnostic means of indisputable accuracy. However, the consideration alone of the fact, that tubercle bacilli can appear in the sputum only after the caseification and breaking down of a tubercle situated near a bronchus or bronchiolus makes it certain that tuberculous changes occur previous to the appearance of the bacillus in the sputum. This is borne out also by the clinical observation of a recognizable stage of tuberculous lung involvement, before bacilli are found in the sputum and the adoption of the term " closed," designating this stage, in opposition to "open," $i$. e., with bacilli found in the sputum, can be recommended for a more general introduction.

From the therapeutic standpoint the diagnosis of pulmonary tuberculosis in its closed stage is of the utmost importance, the chances of permanent recovery diminishing proportionally with the postponement of rational therapeutic measures. These measures, being on the whole nothing but a radical change in the patient's mode of life, will also interfere less with the patients usual occupations, the earlier the diagnosis is made. Hence, also, for this reason, the paramount importance of an early diagnosis. The physician who declines to make a positive diagnosis of tubercu-

*Presented at the Second Annual Meeting of the National Association for the Prevention and Study of Tuberculosis, Washington D. C., May 18, 1905. 
losis on account of the absence of the bacillus in the sputum only, assumes a very grave responsibility, and great caution in this respect cannot be urged sufficiently. Whenever certain signs and symptoms justify a suspicion of the disease, without there being positive evidence, it is wise to instruct the patient carefully as to his mode of life, to watch him closely and to repeat the examination at stated intervals.

As regards the examination, it may be said in general that a close and careful investigation of constitutional as well as local manifestations by the simplest methods will often reveal sufficient evidence for a positive diagnosis. The search for bacilli in the sputum has unfortunately brought about a neglect of these methods.

The history of the patient may or may not contain useful information; it ought to be carefully investigated in every case. Predisposing moments, such as cases of tuberculosis in the family and among intimates or unhygienic mode of life, dusty and confining occupations, must all be taken into consideration, their absence in one case, on the other hand, must not discourage further examination.

The symptoms of incipient tuberculosis will rarely offer anything typical. We may have a very early hoarseness or a condition resembling that of chlorosis or neurasthenia, of bronchitis or dyspepsia. Cough may, or may not, be present. Hemoptysis in the absence of other causes, among all the symptoms, which may be found in the history, is one of the greatest significance. Physical signs are sometimes absent for weeks after the hemoptysis. Vague as all these symptoms may be and characteristic for various morbid conditions, they assume diagnostic value only when considered together with the results of a careful physical examination.

Here inspection, first of all will have to reveal conditions of stature and physical development, which in their deficiencies we know to be indicative if not of the disease itself, at least, of a predisposition to it. Length and weight of body, circumference and degree of expansion of chest, are data of importance, and in their correlation give indication of the bodily condition and state of nutrition. Still we must not depend on finding often the classical habitus phthisicus, the paralytic thorax, if we are to make an early diagnosis of tuberculosis. However, these data as well as the determination of symptoms are of greatest value, if for nothing else but a guidance in subsequent examinations, and for that reason should not be neglected in any case.

Of great importance is the temperature. Even a slight rise of temperature in the afternoon, if other causes can be excluded, ought to arouse invariably our suspicions. Very often this is overlooked, and to shield against such oversight great care in the measuring of the temperature is to be recommended. Whenever possible a two-hourly record for a period of sevoral days ought to be taken, a good thermometer to be placed in the mouth with tightly closed lips and held there for at least five minutes, the patient in a room of even temperature. It will also have to be remembered that in some tuberculous patients the rise of temperature appears only after some exercise, in women before and at the time of menstruation.

The physical examination of the chest by inspection, palpation, percussion and auscultation, if carefully and properly performed, will give more direct evidence than other methods. It may be said here that the newer methods of examination, notably that with the Roentgen rays, cannot, at least, in their present state of development, elaim superiority over the results obtained by the above-mentioned methods.

Attention shall be called here only to a few signs indicating limited lung involvement. On inspection very often a retardation in the respiratory movements over the affected lung portion can be observed, especially over one apex. This retardation is more pronounced in a more recent involvement of the corresponding area of the lung. (Turban.) Foci of greater extent diminish the excursions of the diaphragm of the affected side. This can be demonstrated on the fluorescent screen, but equally well and without apparatus by the observation of the diaphragmatic excursions by means of Litten's shadow.

The vocal fremitus in early pulmonary tuberculosis gives little information. It may be increased or decreased over areas of pleural thickening; over pleuritic exudations it is always diminished.

Painstaking percussion and auscultation of the chest, over all parts and always comparing the two sides, is of the greatest value. The use of the blue pencil for marking the border lines and determining the excursions of the lungs cannot too strongly be urged. Strong percussion, on the whole, should be avoided. Marked dullness is but rarely found over portions of the lungs in incipient tuberculosis; however, the percussion will elicit sometimes a significant retraction of one apex as compared with the other.

In judging the results of the examination by auscultation, it should always be remembered that many of the signs are subject to considerable variation, depending on the time at which the examination is made. Râles which we can easily discover in the morning will regularly be absent during the afternoon. Also on damp and rainy days we will find them when they are absent in dryer weather. Also do we find in women pulmonary signs accentuated at the time of menstruation.

All these circumstances must be considered before a final judgment of the case is given.

Although every portion of the lung (including the lingula over the heart dullness) should be examined with the stethoscope, particular attention will have to be paid to the upper portions of the lungs and also to the lower borders and the axillary regions.

As the earliest auscultatory sign in early pulmonary tuberculosis we can regard the rough and the slightly diminished respiratory murmur. The former must not be confounded with the sharp 
(puerile) respiratory murmur, which is more a sign of increased function than of swelling of the mucosa. Both are vesiculary sounds; the rough character is produced by a succession of murmurs, following each other too rapidly for aural differentiation. Is the succession less rapid, then we speak of an interrupted respiratory murmur, which suggests much coarser changes. Thus the rough murmur changes the character of the respiratory sound, it loses its "smooth" quality and becomes "impure and roughened." (Sahli.) When these adventitious sounds become audible beside the vasicular murmur, then we can speak of râles. The rough murmur is producd by slight inflammatory changes in the bronchioli, the air passing over an uneven surface and through a slightly narrowed lumen. It is principally audible during inspiration over the apices and below the clavicles. This murmur precedes the appearance of râles (not the case, as a rule, with the puerile murmur), and thus is the earliest auscultatory manifestation of tubercular involvement of the air passages. Distinct attention should be paid to it therefore. The appearance of râles over the apices (also in the axillary region) is next to it in importance. Râles indicate catarrhal conditions; with them the intensity of the vesiculary murmur is usually diminished, which is also produced by the more pronounced swelling of the bronchial mucosa. In the earliest stages we hear usually fine crackling râles, they can often only be heard directly after the patient has coughed.

Bronchial respiration we hear but rarely in early tuberculosis; when it appears, we have to deal with a more extensive process. By its localization in the apices and together with other signs it is, of course, pathognomic of consolidation; the same may also be said as regards the other deviation from the normal respiratory murmurs, which are indicative of profound tissue changes, to discuss which does not come within the scope of this report. On the other hand pleuritic friction is often heard at an early period, most frequently in or near the axillary line between the sixth and eighth ribs.

Only passing mention can be made of other diagnostic methods, of which tuberculin is the most important. Although it is well understood that by injection of small doses of tuberculin and by the febrile reaction thus produced in tuberculous individuals, we can diagnose early tuberculosis, the method necessitates great care in its application and an apparatus too complicated for general use, so that it does not lend itself to a more general introduction. The dangers of the preparation in the hands of one well acquainted with the method are very slight, but by applying carefully the other means of observation and examination, a case will rarely be found in which it would add considerably to the information gained.

The fact that certain salts, especially iodine salts, increase catarrhal symptoms and thus make them more perceptible to auscultation, has led to their administration for diagnostic purposes.
For similar reasons as the above stated, a general introduction of this method cannot be recommended.

The examination with $\mathrm{x}$-rays has the drawback of a complicated apparatus. Besides, its value over the other methods has not yet been satisfactorily demonstrated.

Various other methods have been advocated for the early detection of tuberculosis, - inoscopy, sphygmography, sphygmomanometry, serum test, etc., all apt to increase our knowledge of the disease, but of no practical advantage in the everyday diagnosis of so frequent a disease. The careful and painstaking application of the methods well taught and well understood, with the simplest apparatus, but applied with a broad conception of the pathogenesis of the disease, will bring about much earlier diagnoses than are usually made.

\section{LOGICAL AND CLINICAL STANDPOINT.

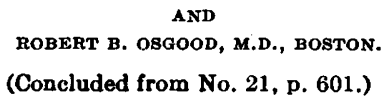

A CONSIDERATION OF THE PELVIC ARTICULATIONS FROM AN ANATOMICAL, PATHO-

CASE I. A patient of Dr. James M. Jackson, thirty years of age, was delivered of a still-born child in the early part of April, 1903. Two years previously a healthy child was born without special difficulty, though with high forceps. After the birth of the first child the patient had been perfectly well, except for an occasional attack of pain in the abdomen, due to a floating kidney. The first part of the recent pregnancy was entirely uneventful. Four months before the child was born, there was an attack of pain due to the floating kidney, and it was necessary for her to remain in bed on her back for two weeks. On getting up she was very weak, especially in the lower part of the back. Walking was difficult, even about the room, and there was a sensation as if breaking down. Walking any distance was practically impossible, because of the discomfort felt about the pelvis. From this time on until delivery, the patient remained quiet at home, and for the last three weeks of the time was kept in bed. The labor started normally. The cervix was taken up, but after this no progress was made. High forceps were tried, but the head was so placed that they could not be applied, and after waiting for some time, version was done, but the child was delivered dead. At the time of the beginning of labor the child was alive, and at the autopsy there was no evidence of any form of disease. Soon after the delivery it was noticed that the symphysis pubes was much separated, so that the finger could be placed between the pubic bones, and when the patient attempted to move the legs or turn over in bed the pubic bones and also the bones at the sacro-iliac articulations moved about. Belts fastened tightly about the pelvis had been used by the attending physician without relief. At the time of the first consultation, no other suggestions were made, but another belt was fitted, and this was continued for a number of days, applied so tightly that the skin was bruised from pressure. A short time after this the patient was turned on her side, so that for the first time it was possible for a thorough examination. At that time the noticeable feature was the absolutely flat back, and instead of the sacrum 